\title{
Adaptive Principal Component Analysis Based Wavelet Transform and Image De-noising for Face Recognition Applications
}

\author{
Isra'a Abdul-Ameer Abdul-Jabbar ${ }^{1,2}$, Jieqing Tan $^{1}$ and Zhengfeng Hou ${ }^{1}$ \\ ${ }^{1}$ School of Computer and Information, Hefei University of Technology, Hefei 230009, \\ People's Republic of China \\ ${ }^{2}$ Computer Science Department, University of Technology, Baghdad, Iraq \\ israa_ameer@yahoo.com,jieqingtan@yahoo.com.cn,houzf@hfut.edu.cn
}

\begin{abstract}
In this paper a novel face recognition approach based on Adaptive Principal Component Analysis (APCA) and de-noised database is produced. The aim of our approach is to overcome PCA disadvantages especially the two limitations of discriminatory power poverty and the computational load complexity, by producing a new adaptive PCA based on single level 2-D discrete wavelet transform using Daubachies filter mode. All face images in ORL database are transformed to JPG file format and are de-noised by Haar wavelet at level 10 of decomposition; the goal is to exhibit the advantage of wavelet over compressed JPG files instead of using origin PGM file format. As a result, our adaptive approach produced good performance in raising the accuracy ratio and reducing both the time and the computation complexities when compared with four other methods represented by standard statistical PCA, Kernel PCA, Gabor PCA and PCA with Back propagation Neural Network (BPNN).
\end{abstract}

Keywords: Face recognition approach, De-noised Database, PCA, APCA, Wavelet Transform

\section{Introduction}

Face recognition has many applicable areas. Moreover, it can be classified into face classification, face identification or gender classification. The most important purposes of face recognition can be applied in many security criteria's such as video content indexing, personal identification in airport, ID card and driver's license, mug shots matching and entrance security. The Principal Component Analysis (PCA) is one of the most common techniques that have been applied in face image recognition and compression. PCA is a statistical method under the wide title of factor analysis. The main goal of PCA is to minimize the large dimensionality of the data space to the small intrinsic dimensionality of feature space, which is needed to describe the data computationally. This is the case when there is a strong correlation between large dimensionality. The works which PCA can do are prediction, redundancy removal, feature extraction and data compression. Because PCA is a conventional technique which can work in the linear domain and can be applied to many application that have linear models such as image processing, pattern recognition, signal processing, system and control theory, and communications [1,2].

The aim of this paper is to produce a novel face recognition approach based on single level 2-D discrete wavelet transform with high accuracy ratio and a little complexity in both terms of time and computational loads; this can be done by finding a specific features in wavelet domain rather than the statistical representation of the covariance matrix in linear domain; and this will lead to higher recognition accuracy rate and faster performance than that of 
standard mathematical PCA. The purpose of this approach is important and can be trusty applied in face identification systems in airport or visa processing and many different security offices since it allows recognizing of human face in real time with high accuracy ratio.

Section 2 describes the related work, Section 3 describes background to PCA eigenface and wavelet transform, Section 4 describes the framework and the structure of the proposed approach, Experiments and results are shown in Section 5, the discussion is described in section 6 and finally the conclusions are described in Section 7.

\section{Related Work}

Face recognition has been an important research area in the computer vision and pattern recognition systems especially in the last two decades. Many researches focused on face identifications based on PCA. The well known eigenface system was developed in 1991 by M.A Turk and P. Alex [3] who gave the basic idea of PCA and produced a real time Eigenfaces system for face recognition using Euclidean distance.

In 1996 Mao [3, 4-8] uses PCA and Nearest Neighbor Classification (NN) with 85\% recognition rate or Minimum Distance Classification (MD) with $77 \%$ recognition rate. While Lawrence [8] find it 83\%. Kyungnam Kim [1] did many experiments with different sets of training image by using well-known face database of AT\&T Laboratories which contains ten different images each of 40 distinct subjects. Although the face recognition results were acceptable, the system only using eigenfaces might not be applicable as a real system.

In 1997, PCA was also applied for dimension reduction for linear discriminate analysis and the algorithm named fisherface was developed. After that, PCA has been extensively employed in face recognition technology [9].

In 2003 also Kaewpijit, et al., [10] found the using of PCA in remote sensing system is expensive and its global nature is time consuming, so that they proposed spectral data reduction in addition to PCA dimension reduction using automatic wavelet decomposition. This is due to the essential characteristic of wavelet transforms keeps the high and the low frequency features, therefore covering all peaks and valleys found in typical spectra. Compared to PCA, for the same level of data reduction, they proved that automatic wavelet reduction precedes comparable classification accuracy for hyper spectral data, while producing substantial computational savings.

In $2004 \mathrm{Ye}$, et al., [11] produced a dimension reduction method, named Generalized Principal Component Analysis (GPCA), the proposed method worked by projecting the images to a vector space that is product of two lower-dimensional vector spaces, the experiments applied on databases of face images with the same storage amount, they found that GPCA is superior to PCA in terms of query precision, quality of the compressed images and computational cost.

In 2009 and 2010 Struc and N. Pave si'c $^{\prime}[12,13]$ designed face recognition toolbox in Matlab 7.0 called it PhD-toolbox, they used real image data and classified face images based on PCA and the nearest neighbor classifier in [14] by using matching score calculation the Mahalanobis cosine similarity 'mahcos' distance in [15] and they find the PCA recognition rate equal to $66.07 \%$ recognition rate for 400 face image.

In 2012 Saurabh, et al., [16] found increasing the number of images and the variety of sample images in the covariance matrix increases the recognition rate however noisy image decrease the recognition accuracy. They found the size of image is not important for a PCA based face recognition system and A. O. Titilayo, et al., [17] modified PCA by performing image projection before applying PCA on the image, named Optimal PCA aimed to reducing the dimension of the covariance matrix involved in PCA. The results of evaluation between both algorithms based on black faces displayed that OPCA and projected combine PCA gave 
recognition accuracies of between $96 \%$ - 64\% and between $95 \%$ - 60\% respectively but not consider the time cost .

Many of researchers also tried enhancing the face recognition system by reducing the face image dimensionality by PCA and classified the face images of PCA with other systems or techniques such as PCA Convolution network produced by Sim [18] with 78\% recognition rate. PCA with back propagation neural network (BPNN) produced by Abul Kashem, et al., [19] they used only 200 images from 400 face images in ORL face database , their system gave $85.5 \%$ accuracy ratio and the execution time reach to 71 seconds.

In our work we proposed enhancement to the PCA method itself instead of support PCA with other networks or techniques and we took in our consideration the raising of accuracy ratio and reducing both of time complexity and computational complexity by producing a novel face recognition approach based on wavelet transform.

\section{Background}

\subsection{Principal Component Analysis}

The main principal of using PCA for face recognition is to extract the large 1-D vector of pixels constructed from 2-D image into the compact principal components of the feature space; this process is called eigenspace projection. Eigenspace is calculated by detecting the eigenvectors of the covariance matrix derived from a set of images (vectors) [2]; we can summarize it in the following steps:

1. Obtain face images $I_{1}, I_{2} \ldots I_{M}$ (training faces).

2. Represent every image $I_{i}$ as a vector $x_{i}$.

3. Compute the average face $\psi=\frac{1}{M} \sum_{i=1}^{M} x_{i}$

4. Subtract the mean face $\varphi_{i}=x_{i}-\psi$

5. Compute the covariance matrix $C=\frac{1}{M} \varphi_{n} \varphi_{n}{ }^{T}=A A^{T}$

6. Compute the eigenvectors $u_{i}$ of $A A^{T}$ :

a. Consider matrix $A A^{T}$ as a $M \times M$ matrix.

b. Compute the eigenvectors $v_{i}$ of $\mathrm{AA}^{\mathrm{T}}$ such that:

$A^{T} A v_{i} \rightarrow \mu_{i} V_{i} \rightarrow A A^{T} A V_{i}=\mu_{i} A v_{i} \rightarrow C u_{i}=\mu_{i} u_{i}$ where $\mu_{i}=A v_{i}$

c. Compute the $\mu$ best eigenvectors of $A A^{T}: \mu_{i}=A v_{\mathrm{i}}$

7. Keep only $K$ eigenvectors by select those vectors with the largest values :

For dimension reduction, $\mathrm{K}$ (where $\mathrm{K}<\mathrm{N}$ ), $\mathrm{N}$ is the number of pixels in the face image, the eigenvector $\mathrm{U}=\left[\mathrm{u}_{1}, \mathrm{u}_{2}, \ldots, \mathrm{u}_{\mathrm{k}}\right]$ corresponding to the largest eigenvalues of the covariance matrix $\mathrm{C}$ are selected as eigenvectors (eigenfaces). For example to reduce the dimension of training samples $\mathrm{Y}$, where $\mathrm{Y}=\left[\mathrm{y}_{1}, \mathrm{y}_{2}, \ldots, \mathrm{y}_{\mathrm{M}}\right]$, We compute $Y=U^{T} X$, and , for class identification a probe image $x_{t}$ is projected on $\mathrm{U}$ to obtain a reduced vector $y_{t=\mathbb{U}^{T}} x_{t}$. A response vector of length $\mathrm{C}, \mathrm{R}\left(\mathrm{x}_{\mathrm{t}}\right)=\left[\mathrm{r}_{1}, \mathrm{r}_{2}, \ldots, \mathrm{r}_{\mathrm{C}}\right]$ is calculated by measuring distances from the probe to the nearest training samples from each class. 
3.1.1. Normalization: A feature is normalized by scaling its values to a small specified range, such as 0 to 1 . The normalization step is applied to prevent features with large ranges from superiority features with smaller ranges. Min-max normalization has a linear transformation on the original data. Assume the min and max are the minimum and the maximum values for feature. Min-max normalization transforms the value $v$ of $A$ to $v$ ' in the range [newmin, newmax] by computing [2]:

$$
\mathrm{v}^{\prime}=(\mathrm{v}-\min (\mathrm{a}))(\max (\mathrm{a}) \times \min (\mathrm{a})) \times\left(\text { new }_{\max }-\text { new }_{\min }\right)+\text { new }
$$

3.1.2. Eigenface Matching: Let $X$ and $Y$ are two feature vectors of spectral eigenface where, $x i \in X, y i \in Y, i=1 \ldots n$, and to calculate the degree of association, the correlation distance is defined as [2]:

$$
R=1-r
$$

This is given by the formula [2]:

$$
r(x, y)=\frac{\sum\left(x_{i}-\bar{x}\right)\left(y_{i}-\bar{y}\right)}{\sqrt{\sum\left(x_{i}-\bar{x}\right)^{2} \sqrt{\sum\left(y_{i}-\bar{y}\right)^{2}}}}
$$

Where: $\bar{x}$ is the mean of vector $\mathrm{X}$, and $\bar{y}$ is the mean of the vector Y.

The correlation distance determines the original query sample; it is suitable to verify the input face image by a pre-defined threshold value $\mathrm{T}$. If the value $\mathrm{R}$ is smaller than threshold $\mathrm{T}, \mathrm{R}<\mathrm{T}$, then the holder of the query sample is declared as a subject $\mathrm{X}$. Otherwise, the query sample is classified as a forged subject.

\subsection{2-D Discrete Wavelet Transform}

The discrete wavelet transform (DWT) is a mathematical technique that decomposes a signal into a set of multi-scaled wavelets. Since DWT has many useful characteristics can be applied to various scientific applications, such as those of image and video processing, speech processing, numerical analysis, and pattern recognition [20, 21].

The wavelet is used to decompose the image into subbands of wavelet transform each one with different coefficients. An image, which is a two dimensional signal, is decomposed using the two dimensional wavelet tree decomposition algorithm. The original image is processed along the $X$ and $Y$ direction by $L_{0}-D$ and $H_{I^{-}} D$ filter bank which is the row representation of the original image. It is decomposed row-wise for every row using one dimension decomposition algorithm to produce two levels of Low $(L)$ and High $(H)$ components approximation. The term $L$ and $H$ refer to whether the processing is low pass or high pass filters. Because of the down sampling process that is performed on the $\mathrm{L}$ and $\mathrm{H}$ image gives matrices are rectangular of size $(N \times N / 2)$ matrices which is again transposed and decomposed to obtain four $(N / 2 \times N / 2)$ square matrices. The down sampling that performs on these matrices will generate $L L, L H, H L$, and $H H$ components. Each of these components corresponds to four different wavelet sub band. The LL component called the approximation function component decomposed to obtain further details of the image; the other wavelet components called (CA, CD horizontal, $\mathrm{CD}$ vertical, $\mathrm{CD}$ diagonal) can also be decomposed further $[22,23]$.

To find the Correlation implementation using wavelet transform, Let X, and Y be data sets such that, correlations based WT is defend as: take WT of X, and WT of Y, multiply one resulting transform by the complex conjugate of the other, and inverse transform the result product such as [2]: 


$$
\operatorname{Corr}(X, Y)=I W T(W T(X) \times W T(Y)
$$

\section{The Framework of the Adaptive Approach}

The proposed approach has the following block diagram as shown in Figure 1:

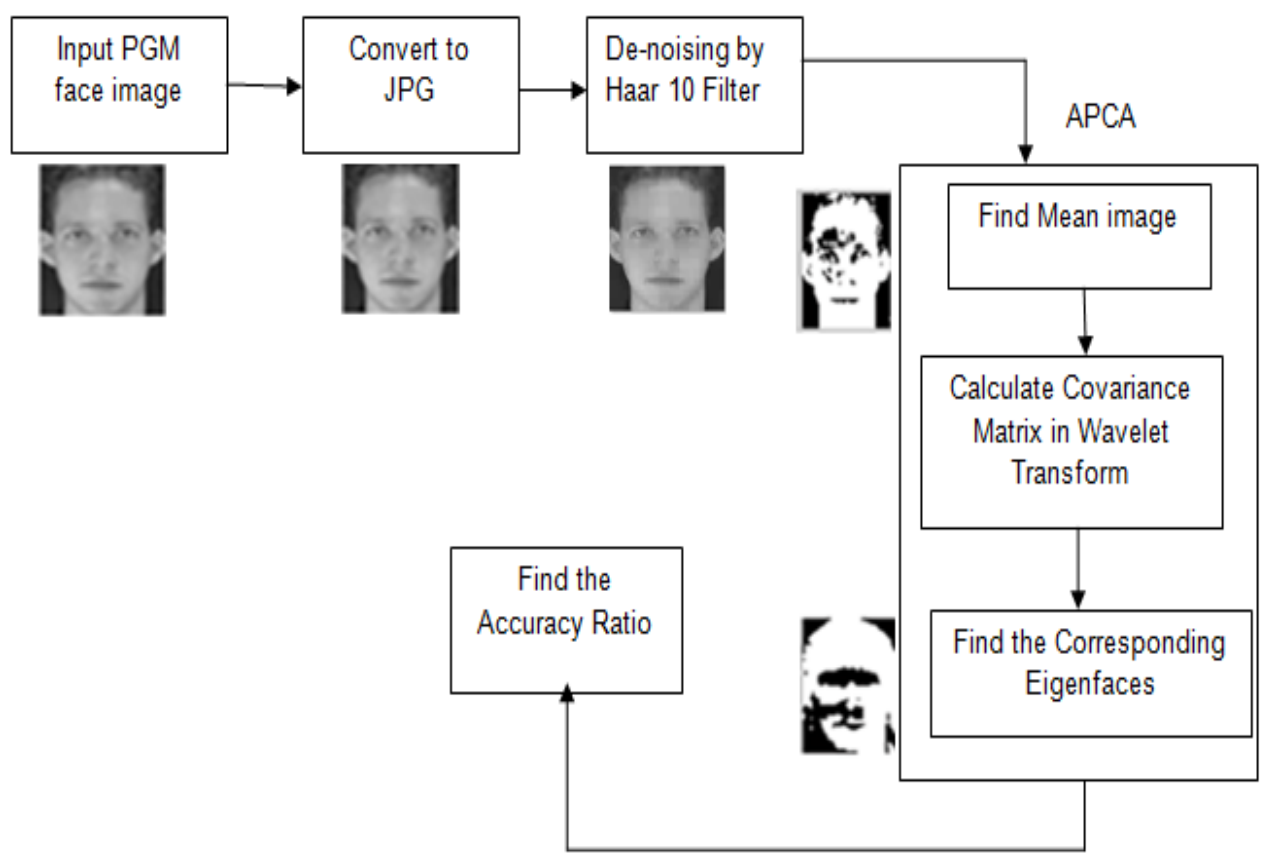

Figure 1. The Adaptive Approach Block Diagram

\subsection{The Proposed APCA}

The proposed idea of applying the wavelet transform in the implementation of Eigenface is done by the using of a single-level two-dimensional wavelet decomposition in the implementation of the covariance matrix, as an alternative of conventional ideas of converting the intensity of the image face data into the spectral domain, followed by applying the Eigenface. The proposed idea is called as Adaptive Principles Components Analysis (APCA) based Wavelet Transform.

The covariance matrix can be computed by using the wavelet transform (WT) as follows: WT the two datasets, multiply one resulting transform by the complex conjugate of the other, and inverse transform the product [9].

Here are the steps to computing these Eigenfaces:

1. Obtain face images $I_{1}, I_{2} \ldots I_{M}$ (training faces).

2. Represent every image $I_{i}$ as a vector $x_{i}$.

3. Compute the average face $\psi=\frac{1}{M} \sum_{i=1}^{M} x_{i}$

4. Subtract the mean face $\varphi_{i}=x_{i}-\psi_{i}$

5. Compute the covariance matrix using a single-level two-dimensional wavelet with Daubechies filters mode (db1, db2, db6, db10).

$$
C=I W T\left(W T(\varphi) W T\left(\varphi^{T}\right)\right)=A A^{T}
$$


a. [cAl,cH1,cVl,cDl] $=d w t 2(\varphi$, 'Daubechies filter type')

b. $[c A 2, c H 2, c V 2, c D 2]=d w t 2(\varphi$, 'Daubechies filter type')

c. $C=I d w t 2(c A 1 \times c A 2, c H 1 \times c H 2, c V 1 \times c V 2, c D 1 \times c D 2$, 'Daubechies filter type')

6. Compute the eigenvectors $u_{i}$ of $A A^{T}$ :

a. Consider matrix $A A^{T}$ as an $M \times M$ matrix.

b. Compute the eigenvectors $v_{i}$ of $A A^{T}$ such that:

$A^{T} A v i \rightarrow \mu_{i} V_{i} \rightarrow A A^{T} A V_{i}=\mu_{i} A v_{i} \rightarrow C u_{i}=\mu_{i} u_{i}$ Where $\mu_{i}=A v_{i}$

c. Compute the $\mu$ best eigenvectors of $A A^{T}: \mu_{i}=A v_{i}$

7. Keep only the largest $K$ eigenvectors of covariance matrix $C$.

\subsection{The Proposed De-noised Database by Haar Wavelet Filter}

The original Image database consists of a collection of faces taken between April 1992 and April 1994 at the Olivetti Research Laboratory (ORL) in Cambridge, United Kingdom. This collection included 10 different images of 40 distinct subjects. The images were picked at different times with varying luminance and facial expressions "open/closed eyes, smiling/ non-smiling "and facial details "glasses/no-glasses. All the images are taken against a dark homogeneous background and the subjects were in up-right, frontal position "with acceptance for some side movement". The image files were in Portable Gray Map (PGM) format, with a size 92x112, 8-bit grey levels [24, 25].

In our work all images are transformed to JPG format and de-noised by Haar wavelet at level 10 of decomposition, the reason that we transform the whole image database to JPG format that we seen from previous experiments that the de-noised JPG image gave the highest recognition rate when we applied it on the original PCA, since by this experiment we evaluate the effect of JPG, BMP, TIFF, PNG and GIF file formats on raising the face recognition ratio in some face recognition methods such as PCA, Linear Discriminate Analysis (LDA), Kernel PCA (KPCA) and Kernel Fisher Analysis (KFA). The following Steps were done on the original AT\&T ORL database:

1. Load original faces database of PGM file format.

2. Convert all face images to JPG file formats

3. Choose Haar wavelet filter with ten level of decomposition. Then compute the 2DDWT of the noisy image.

4. Threshold the non-LL subbands.

5. Perform the inverse wavelet transform on the original Approximation LL-subband and the modified non-LL subbands for each face image in the database.

6. Keep the new JPG de-noised database.

Figure 2 shows a sample of images from original ORL database and Figure 3 shows a sample of image de-noised by Haar wavelet at level 10 of decomposition. 


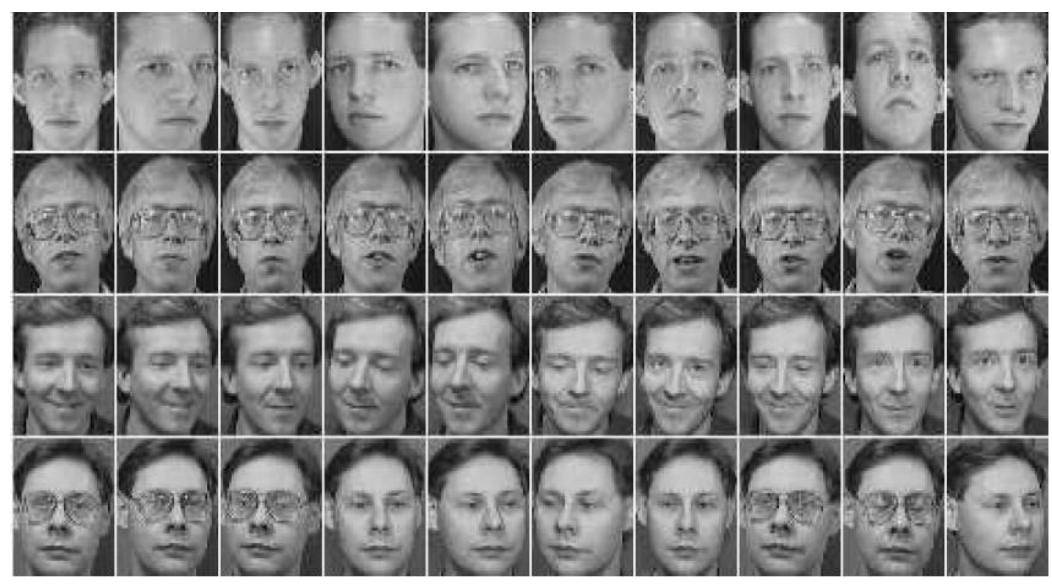

Figure 2. Sample of Face Images from the ORL Face Database

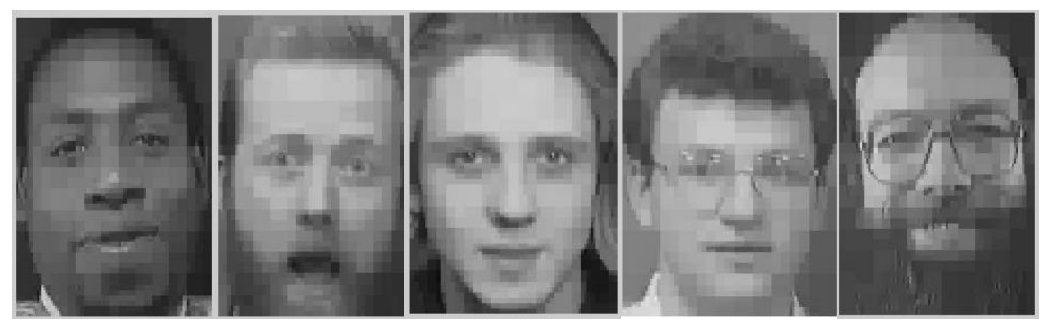

Figure 3. Sample of Faces de-noised by Haar Wavelet at Level 10 of Decomposition

\section{Experiments and Results}

The proposed APCA is tested using a set of faces in the proposed database. The number of faces in the database is 400 images as in the origin ORL database; in our experiments we take 200 images (5 for each individual) as test image and the other 200 image as training Set to find the matching accuracy ratio for APCA and compared it with the original matching accuracy ratio of PCA on ORL database, Table 1 shown the result of APCA when the covariance matrix implemented in discrete wavelet transform with different types of filters mode ( $\mathrm{db} 1$ or Haar, db2, db6 and db10) filters. The experiments also tested the original PCA and the proposed APCA with original ORL database of PGM image file format and with JPG file formats both tests is done before and after the Haar wavelet de-noising process at level 10 of decomposition implemented on these databases.

Table 1. Accuracy Ratio of the Original PCA and the Adaptive PCA with and Without de-noising

\begin{tabular}{|l|c|c|c|c|c|c|}
\hline \multirow{2}{*}{ Database type } & \multirow{2}{*}{\begin{tabular}{c} 
Std. \\
\cline { 3 - 7 }
\end{tabular}} & PCA & $d b 1$ & $d b 2$ & $d b 6$ & $d b 10$ \\
\hline \multirow{2}{*}{$\begin{array}{l}\text { Without } \\
\text { de-noising }\end{array}$} & PGM & $77 \%$ & $76 \%$ & $77.5 \%$ & $77.5 \%$ & $77 \%$ \\
\cline { 4 - 7 } & JPG & $82 \%$ & $83.5 \%$ & $83 \%$ & $83 \%$ & $84 \%$ \\
\hline $\begin{array}{l}\text { After } \\
\text { de-noising }\end{array}$ & PGM+WT & $74.5 \%$ & $75 \%$ & $75.5 \%$ & $75.5 \%$ & $75.5 \%$ \\
\cline { 2 - 7 } & JPG+WT & $83.5 \%$ & $84.5 \%$ & $85 \%$ & $84.5 \%$ & $85.5 \%$ \\
\hline
\end{tabular}


Table 2. Comparison in Accuracy Ratio and Computation Time among Different Methods on Original PGM ORL Database and our Approach (APCA on JPG deNoised ORL Database)

\begin{tabular}{|c|l|l|l|l|}
\hline Method & Method Used & $\begin{array}{c}\text { No. of } \\
\text { Image }\end{array}$ & $\begin{array}{c}\text { Time of } \\
\text { execution in } \\
\text { second }\end{array}$ & Accuracy \\
\hline Standard PCA & PCA & 200 & 5.6043 & $77 \%$ \\
\hline Struc[12-13] & PCA + Mahcos & 200 & 7.8519 & $76 \%$ \\
\hline Struc[12-13] & Kernel PCA & 200 & 6.3767 & $45 \%$ \\
\hline Struc[12-13] & Gabor PCA & 200 & 87.5211 & $70 \%$ \\
\hline Abdul kashem [19] & PCA+BPNN & 200 & 71 & $85.5 \%$ \\
\hline Our approach & APCA & 200 & 5.2903 & $85.5 \%$ \\
\hline
\end{tabular}

In the Table 2, we computed the time of calculating the accuracy ratio to 200 face images in Standard PCA, PCA using Mahalanobis cosine similarity, Kernel PCA methods and Gabor PCA in [12-13] and compare it with the time of implementation of APCA when the same number of images is used from ORL database. And as shown our proposed approach (the adaptive PCA using db10 with de-noised database) is better than the other methods because it's produced high accuracy ratio equal to $85.5 \%$ which is equivalent to the result of PCA with back propagation neural network (BPNN) in [19] but reduced the time to only 5 seconds rather than $71 \mathrm{sec}$.

\section{Table 3. Time Computation of the Original PCA and the Proposed APCA with Different Daubechies Wavelet Filters on the Proposed Database}

\begin{tabular}{|c|c|c|c|c|c|}
\hline \multirow{2}{*}{ Method } & PCA & \multicolumn{4}{|c|}{ of ORL on proposed database } \\
\cline { 3 - 6 } & database & $d b 1$ & $d b 2$ & $d b 6$ & $d b 10$ \\
\hline $\begin{array}{l}\text { Times in } \\
\text { seconds }\end{array}$ & 5.6043 & 4.5242 & 4.6592 & 4.9871 & 5.2903 \\
\hline
\end{tabular}

From the result of Table 1 we can observe that the transformation of face images from PGM to JPG file formats increased the matching accuracy ratio of both the PCA (82\%) and the proposed APCA (84\%), and the implementation of Haar10 wavelet failed to raise the accuracy ratio for the original PCA and its reduced from (77\% to $74.5 \%)$, but it produced the highest result with our proposed database on both the PCA and APCA to give the best result when the covariance matrix is computed in discrete wavelet transform by db10 filter mode to reach to $(85.5 \%)$ ).In addition to the increasing of the accuracy ratio of our proposed methods it is also highly optimized for precision computations and allows fast computing even for reasonably computer specification of (Core i3, 2.40GHz) as shown in Table 2 and Table 3.

To explain the difference in feature extraction process between the proposed APCA and PCA, we take a sample of 20 images from the proposed database as shown in Figure 4, and the mean for the two methods are shown in Figure 5. A min-max normalization is applied firstly to normalized the feature vector in range $[0,1]$, then the covariance matrix is computed in APCA is $(92 \times 112 \times 20)$ and the size of the projected image is $20 \times 20$ since there is 20 eigenface vector with 20 eigenvalue (one value for each sorted eigenvector) as shown in Figure 6 while in PCA we found it has the same covariance matrix but the projected image is $19 \times 20$ (number of image-1 with 19 eignvalue), Figure 7 shows the eigenspace of origin PCA when applied on proposed database. 
For eigenface matching, a correlation distance is computed between two spectral eigenface feature vectors, and it is easy to verify the input pattern using specific threshold, in our proposed PCAWT we used the same threshold that used by Mohed Zubair Saifi where ( $\mathrm{T}>$ $0.5 \times 10^{8}$ ) for code written in PCA based face recognition system for feature extraction process, and for the calculation of the accuracy ratio we used $(\mathrm{T}<0.9)$ for both standard PCA and APCA, then the accuracy ratio is compared as shown in Table 1.

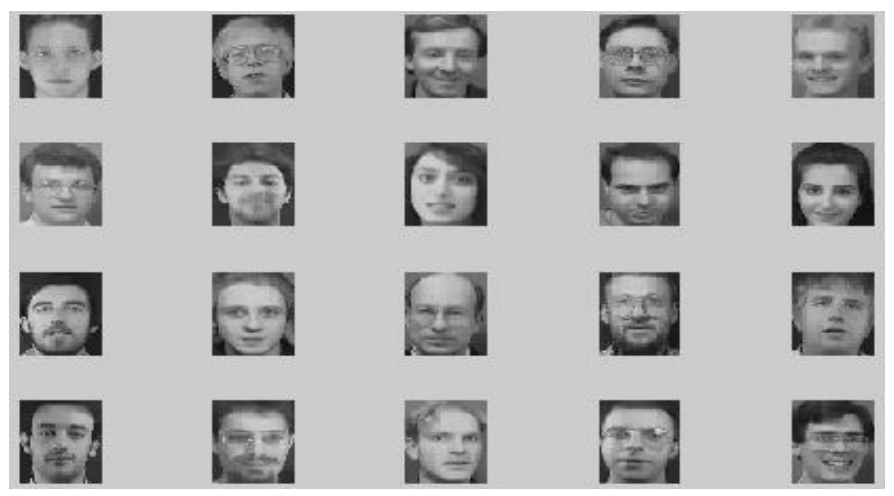

Figure 4. Sample of Face Images from our De-Noised Database (JPG+WT)

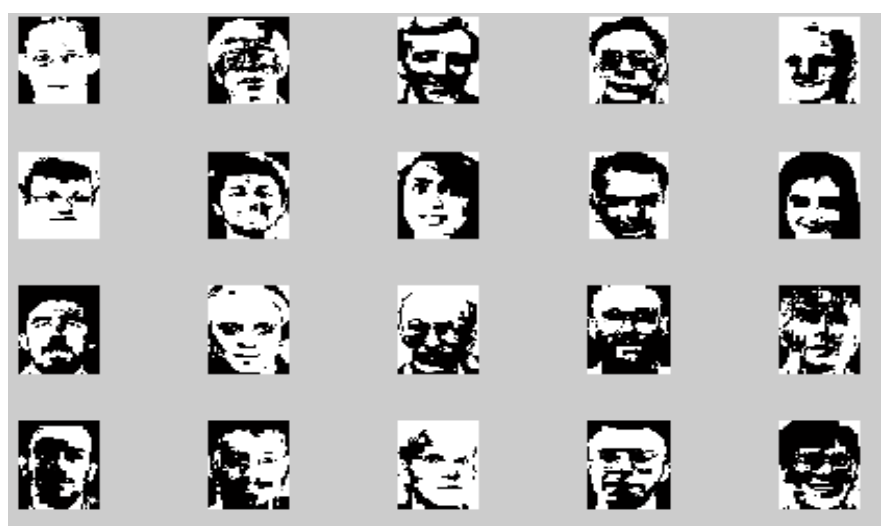

Figure 5. Mean Faces

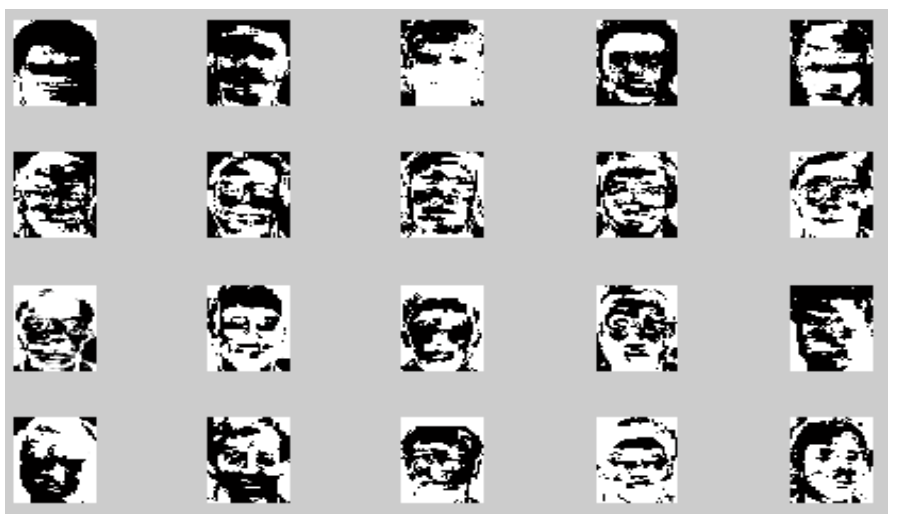

Figure 6. 20 Eigenfaces in APCA for 20 Faces from our Proposed Database 


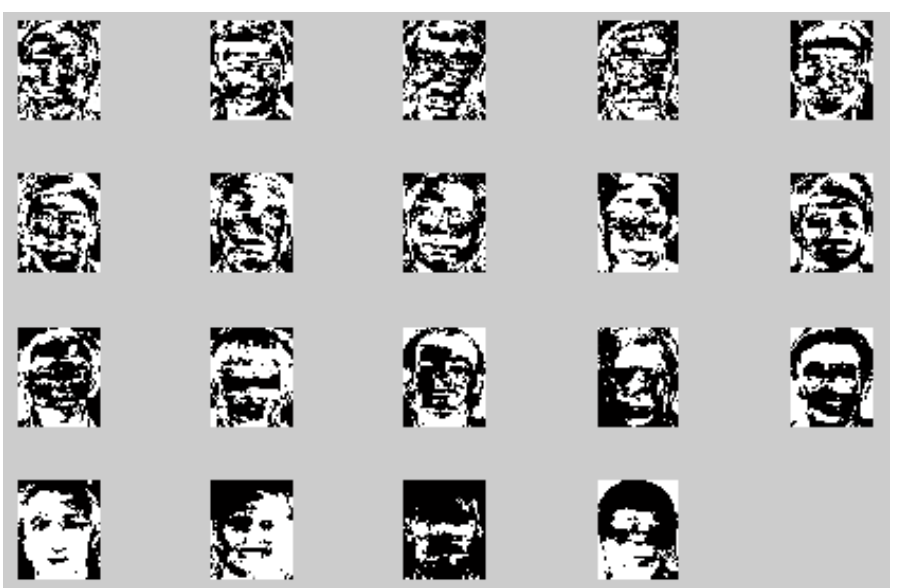

Figure 7. 19 Eigenfaces in PCA for 20 Faces from PGM ORL Database

\section{Discussion}

In this Section we will discuss the process of feature extraction based wavelet domain, since the wavelet transform are very important tools, which are widely used in feature extraction in many fields such as image compression and de-noising applications. Wavelets are mathematical functions which give different frequency components of data. The advantages of WT are good in both time and frequency localizations. In our work we exhibit these advantages in both image frequency analysis and image decomposition and we applied them on PCA because of the following reasons [26]:

- The resolutions of the images can be reduced by decomposing an image using wavelet transform and working on a lower resolution image, this will reduce the computational complexity.

-WT provides local information in both spatial domain and frequency domain.

Feature extraction is the most important Step in face recognition. The main aim of feature selection is to pick an optimal subset of features from a given space; this will lead to high classification performance. In the eigenspace, all the eigenvectors are not equally informative. We found as in [27] that different eigenvectors include different kind of information and the appearance of the eigenvalues order specify the importance of eigenvectors, this order is not always appropriate to describe the data. For example, the first eigenvectors seem to include lighting while other eigenvectors seem to include features such as glasses or moustaches. Although many of the eigen features are very common for face recognition, they might actually baffle the classifier in other applications. In our work, we used the Daubechies wavelets, the reason is, because Daubachies filters are widely used in solving a broad range of problems, for example self-similarity properties of a signal or fractal problems, signal discontinuities for choosing of the eigenvectors and to select a good subset of eigen features in order to enhance face recognition performance, as a result reduces the computation complexity and also increases the recognition rate.

\section{Conclusions}

This paper subjected a new face recognition approach based on adaptive PCA in wavelet domain and de-noised database by Haar wavelet filter. In our approach, we investigated the ability of implementing the eigenfaces in the frequency domain by using wavelet transform 
with Daubechies filter mode as a method to recognize the face in human identification system. The conclusions can be summarized as follow:

- The using of Haar wavelet at level 10 over JPG rather than PGM file format of face images has the main reason to enhance the matching accuracy ratio of face images when compared to the original PCA on original ORL database because the flexibility of wavelet transform over compressed file images like the JPG file format, and Daubachies 10 is proved to be the best filter mode; since one of the major advantages of wavelet based eigenfaces recognition scheme is the ease of implementation.

- Recognizing face images in real time systems with high accuracy performance can be worthily used for security applications such as human identification systems in airports, visa processing, ID card verification, driver license, police office for verifying criminals and monitoring systems.

- Our proposed de-noised database not only contributed in getting high accuracy ratio in our adaptive PCA, but it is also contributed in raising the accuracy ratio in some methods that used in our experiments such as standard PCA, PCA+Mahcos, KPCA and Gabor PCA to reach to $82 \%, 80 \%, 52 \%$ and $90 \%$ respectively, which is higher than the accuracy ratio indicated in Table 2 when these methods implemented on original ORL database.

\section{Acknowledgments}

This work is supported by the NSFC-Guangdong Joint Foundation (Key Project) under Grant No.U1135003 and the National Natural Science Foundation of China under Grant No.61070227.

\section{References}

[1] K. Kim, "Editor, Face recognition using principle component analysis", International Conference on Computer Vision and Pattern Recognition, USA, (1996).

[2] H. M. Salman, "Principal component analysis based wavelet transform", Engineering and Technology Journal, vol. 30, no. 9, (2012).

[3] M. Turk and A. Pentland, "Eigenfaces for recognition", Journal of Cognitive Neuroscience, vol. 3, no. 1, (1991).

[4] P. Miller and J. Lyle, "The effect of distance measures on the recognition rates of PCA and LDA based facial recognition", Digital Image Processing, (2008).

[5] A. Pentland, B. Moghaddam and T. Starner, "View based and modular eigenspaces for face recognition", In Computer Vision and Pattern Recognition. Proceedings CVPR'94., 1994 IEEE Computer Society Conference on ,IEEE, Seattle, WA, (1994) June.

[6] M. Satone and G. K. Kharate, "Selection of Eigenvectors for Face Recognition", International Journal of Advanced Computer Science and Applications, vol. 4, no. 3, (2013).

[7] T. Boult, "Robust distance measures for face-recognition suporting revocable biometric tokens", Proceeding of 7th International Conference in the Automatic Face and Gesture Recognition FGR, IEEE, (2006) April 26, Southampton.

[8] S. Lawrence, C. L. Giles and Tsoi, A. C, “Convolutional neural networks for face recognition", In Computer Vision and Pattern Recognitio, 1996. Proceeding CVPR'96, 1996 IEEE Computer Society Conference on, IEEE, CA, (1996), June, San Francisco.

[9] H. Zhao, P. C. Yuen and J. T. Kwok, "A Novel Incremental Principal Component Analysis and Its Application for Face Recognition", IEEE Transaction on Systems, Man, and Cybernetics-part B: Cybernetics, vol. 36, no. 4, (2006).

[10] S. Kaewpijit, J. Le Moigne and T. El-Ghazawi, "Automatic reduction of hyperspectral imagery using wavelet spectral analysis. Geoscience and Remote Sensing”, IEEE Transactions, vol. 41, no. 4, (2003).

[11] J. Ye, R. Janardan and Q. Li, "GPCA: an efficient dimension reduction scheme for image compression and retrieval", KDD '04 Proceedings of the tenth ACM SIGKDD international conference on Knowledge discovery and data mining, (2004), New York, NY, USA.

[12] Struc and N. Pavessi'c, "The complete gabor-fisher classifier for robust face recognition," EURASIP Advances in Signal Processing, doi $=10.1155$ / $2010 /$ 847680, (2010). 
[13] Struc and N. Pave si'c, "Gabor-based kernel partial-least-squares discrimination features for face recognition," Informatica (Vilnius), vol. 20, no. 1, (2009).

[14] M. Teixeira D. Bolme, R. Beveridge and B. Draper, "The CSU Face Identification Evaluation System: Its Purpose, Features and Structure", International Conference on Vision Systems, (2003).

[15] M. Basseville, "Distance measures for signal processing and pattern recognition", Signal processing,vol. 18, no. 4, (1989).

[16] S. P. Bahurupi and D. S. Chaudha, "Principal Component Analysis for Face Recognition", International Journal of Engineering and Advanced Technology, vol. 1, no. 5, (2012).

[17] O. T. Adedeji , E. O. Omidiora, S. O. Olabiyisi and A. A. Adigun, "Performance Evaluation of Optimised PCA and Projection Combined PCA methods in Facial Images", J. of Computations and Modelling, vol. 2, no. 3, (2012).

[18] T. Sim, R. Sukthankar, M. Mullin and S. Baluja, "Memory-based Face Recognition for Visitor Identification", In Automatic Face and Gesture Recognition, 2000. Proceedings. Fourth IEEE International Conference on, IEEE, (2000) March 28-30, Grenoble.

[19] M. Abul Kashem, Md. Nasim Akhter, Shamim Ahmed and Mahbub Alam, "Face recognition system based on Principal Component Analysis(PCA) with Back Propagetion Neural Nework(BPNN)", Canadian Journal on Image Processing and Computer Vision, vol. 2, no. 4, (2011).

[20] Z. Li, W. Shi and K. Wang, "Xiaowei Sun, Zhi Zhong”, New Correlation Analysis Method for Nonstationary Signal, Wseas Transaction on Information Science and Application, vol. 5, no. 12, (2008).

[21] S. C. Chen and C. C. Wu, "Editors. An Architecture of 2-D 3-level lifting-based discrete wavelet transform. Proceeding of the 2002 VLSI Design/ CAD Symposium, (2002) August.

[22] M. Sifuzzaman, M. R. Islam and M. Z. Ali, "Application of wavelet transform and its advantages compared to Fourier transform," Journal of Physical Sciences, vol. 13, (2009).

[23] O. O. Khalifa, M. Yusuf and S. Gagula, "Face Recognition Using PCA Wavelet Decomposition", (2001).

[24] R. Gross, S. Li and A. Jain, "Editors. Handbook of Face Recognition", Springer Verlag (2005).

[25] P. J. Phillips and E. M. Newton, "Editors. Meta-analysis of face recognition algorithms", In Automatic Face and Gesture Recognition, 2002. Proceedings. Fifth IEEE International Conference on,IEEE, (2002) May 2021, Washington, DC,USA.

[26] R. C. Gonzalez, R. E. Woods and S. L. Eddins, "Editors. Digital image processing using Matlab", China Edition Published by Pearson Education Asia LTD (2009).

[27] M. Satone and G. K. Kharate, "Selection of Eigenvectors for Face Recognition", International Journal of Advanced Computer Science and Applications, vol. 4, no. 3, (2013).

\section{Authors}

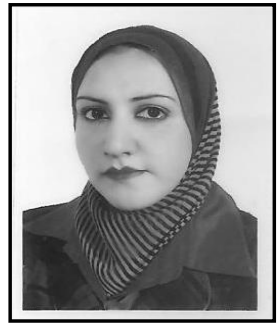

Isra'a Abdul-Ameer Abdul-Jabbar, received her B.S. degree in 2003 and M.Sc. degree in 2006 in Computer Science both were from the Department of Computer Science, University of Technology, in Baghdad, Iraq. She is now a PhD. candidate at the School of Computer and Information in Hefei University of Technology, in Hefei, China. Her research interests include face Recognition Systems and Image Processing.

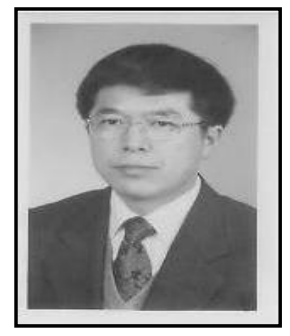

Jieqing Tan, received his Ph.D. degree in Computational Mathematics from Jilin University, China, in 1990, and worked in Fachbereich Mathematik, Universitaet Dortmund, Germany as a Postdoctoral from 1992 to 1993 . He is a Professor at Hefei University of Technology and Director at the Institute of Applied Mathematics from 1996 and Supervisor of doctoral students from 1998. His research interests include nonlinear numerical approximation, scientific computing, computer aided geometric design, computer graphics and digital image processing. 